\title{
Impact of COVID-19 on paediatric and OBGYN residency training in Singapore
}

\section{Dear Editor,}

The ongoing COVID-19 pandemic is likely to have far-reaching implications on residency training under the competency-based residency system, Accreditation Council for Graduate Medical Education I (ACGME-I). COVID-19 caused considerable disruption due to the longer infectious period and greater asymptomatic transmissibility. ${ }^{1}$ This study aims to identify the impact of COVID-19 on paediatrics, and obstetrics and gynaecology (OBGYN) residency in KK Women's and Children's Hospital (KKH), Singapore.

A qualitative phenomenological approach was used to conduct semi-structured individual interviews involving 15 paediatrics and 15 OBGYN residents. These interviews were conducted either in person or via telecommunication platforms between March 2020 and April 2020. Residents were selectively sampled to ensure diversity in personal roles and social background such as parents, marriage status and years in residency (Table 1A). The conceptual framework of this study was structured with reference to the studies from previous pandemics. $^{2-7}$ Data saturation ${ }^{8}$ was reached after a total of 30 residents, with 15 residents from each programme interviewed.

The interview guide consisted of a standardised set of open-ended questions focusing on the 3 major domains (Table 1B) derived from literature review of studies on SARS outbreak and H1N1 pandemic:2-7 training, clinical environment and psychosocial impact. Thematic analysis was performed to identify emerging themes through descriptive phrases from the participants (Table 1C). To reduce potential bias, the interviews were recorded and each transcript was reviewed for themes by at least 2 authors independently, who were not involved in the interview process and were from the other residency programme.

From the transcripts of the interview, the participants described key aspects of their clinical training directly influenced by COVID-19 pandemic (Theme 1). With residents being redeployed to cover the isolation wards, rotations to subspecialties had been reduced. Non-urgent clinic visits and elective surgeries were rescheduled. Residents rotated to external hospitals were arranged to remain in these external postings, delaying the resumption of their core postings. Collectively, these had affected their year-on-year training progression. Residents were also unable to attend teaching sessions due to the insufficient manpower in the wards. A multitude of activities designed for learning were reduced, including grand ward rounds, clinical audit meetings, conferences, bedside tutorials, multidisciplinary team meetings, workshops and elective postings. Some of these courses are compulsory requirements for residency, hence their deferment would disrupt residency progression. Cancellation of exit examination has affected career progression, resulting in low morale among senior residents. The delivery of clinical education was challenging as didactic teachings were conducted over teleconferencing platforms such as Zoom. Virtual teaching may not be effective due to the lack of personalised contact and classroom participation. Moreover, videoconferencing was unable to replace practical hands-on training sessions. Thus, the quality of learning from clinical day-to-day work was perceived to have been significantly compromised. To practise social distancing, residents were no longer able to observe a senior colleague in a clinic session to learn specific counselling skills.

Changes in the clinical environment have significantly reduced the working efficacy of the residents (Theme $2)$. The hospital created a set of infection control protocols to provide guidance in suspect and confirmed cases' management. As these protocols were constantly evolving, it was challenging for residents to keep up. Respondents expressed the frustration of information overload as multiple emails on protocol updates were received regularly. The experience was aggravated with protocols that were unfamiliar in their normal daily practice. While personal protective equipment (PPE) and COVID-19 test kits provided safety assurance, donning PPE required additional time, especially during emergency situations such as acute resuscitation. Occasionally, strict protocols for PPE may not be stringently adhered to, raising concerns over the potential exposure to COVID-19. Additionally, the physician-patient relationship was often hampered by the usage of PPE as non-verbal communication, such as subtle cues during clinical interactions, was significantly impacted. Specifically, the fear that children experience when seeing healthcare staff in PPE greatly affected the clinical experience of the paediatrics residents, reducing 
Table 1. Design of the study

(A) Profile of the residents, with baseline demographics and year of residency training

\begin{tabular}{|c|c|c|}
\hline & OBGYN residency $(n=15)$ & Paediatrics residency $(n=15)$ \\
\hline \multicolumn{3}{|l|}{ Demographics } \\
\hline Age, years, mean $\pm \mathrm{SD}$ & $31.8 \pm 3.6$ & $31.5 \pm 3.0$ \\
\hline \multicolumn{3}{|l|}{ Sex } \\
\hline Male, no. (\%) & $3(10.0)$ & $7(23.3)$ \\
\hline Female, no. $(\%)$ & $12(40.0)$ & $8(26.7)$ \\
\hline \multicolumn{3}{|l|}{ Nationality } \\
\hline Singaporean, no. (\%) & $14(46.7)$ & $11(36.7)$ \\
\hline Others, no. (\%) & $1(3.3)$ & $4(13.3)$ \\
\hline \multicolumn{3}{|l|}{ Marital Status } \\
\hline Married, no. (\%) & $7(23.3)$ & $7(23.3)$ \\
\hline \multicolumn{3}{|c|}{ Year of residency training, no. $(\%)$} \\
\hline Year 1 & $1(3.3)$ & $3(10.0)$ \\
\hline Year 2 & $2(6.7)$ & $2(6.7)$ \\
\hline Year 3 & $2(6.7)$ & $2(6.7)$ \\
\hline Year 4 & $3(10.0)$ & $1(3.3)$ \\
\hline Year 5 & $2(6.7)$ & $4(13.3)$ \\
\hline Year 6 & $5(16.7)$ & $3(10.0)$ \\
\hline
\end{tabular}

Percentage is that of total participants

OBGYN: obstetrics and gynaecology: SD: standard deviation

(B) Standardised set of interview questions

\section{Interview Questions}

\section{Key questions}

1. Can you share how things at the workplace are different now compared to peace time? How do you feel about these changes? (training/clinical environment)

2. Can you share how things in your personal life have changed since the start of the pandemic? How do you feel about these changes? (psychosocial)

3. What would you like the hospital and residency programme to do (or to do differently) for residents like yourself in this time?

Probing question examples (if specific research areas have not been covered in key questions):

1. How do you feel about having to work in a high-risk area?

2. How do you feel about the residency training that you are experiencing now?

3. How do you feel about the work assignments (e.g. rosters, job scope) that you are currently performing?

their work satisfaction. Decrease in the number of doctors participating in ward rounds also resulted in a lack of continuity of care. The issue of burnout has also been a major concern. With the greater division of manpower to cover both the general and isolation wards, the workload was significantly higher for each resident. Some respondents reported an increased number of overnight calls, sometimes up to 3 times in a week.

The impact of COVID-19 on the resident's psychosocial well-being may directly affect their performance 
(C) Emerging themes through thematic analysis of the descriptive phrases from the participants

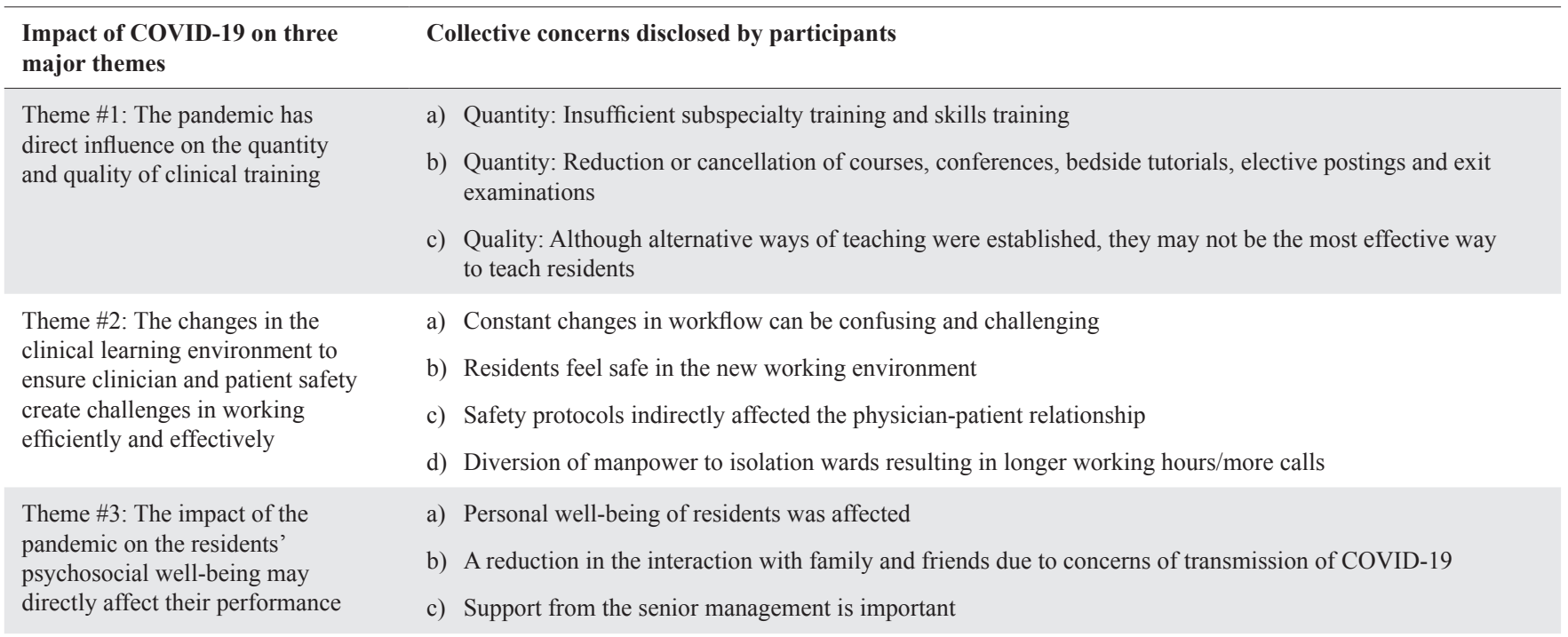

(Theme 3). While most respondents felt empowered to contribute to society and the community at large, some experienced low morale and motivation due to leave cancellations, concern over residency promotion, repeated deployment for isolation ward shifts, and the impact on work-life balance. The residents expressed fears that this may lead to burnout and being underappreciated. There were also concerns of being stigmatised on being tested positive for COVID-19. Additionally, residents were concerned about possible transmission of the disease to their family members and friends, especially those with young children. To prevent the potential spread of COVID-19, residents had been refraining from meeting their friends and extended families. Some residents made care arrangements for their children to minimize the risk of exposure. While most family members were understanding and supportive, one respondent recounted that she was advised not to work in the high-risk area if given the choice. Lastly, respondents expressed concerns about the lack of information and transparency to their training continuity as well as work protocols. The issues of paucity of input from the residents, and lack of engagement in matters pertaining to changes in policies and rosters were raised. The residency programmes had not provided clarity or reassurances on the impact of changes in the training on their individual progression. Although the efforts of programme executives in gathering feedback and arranging alternative modes of teaching were appreciated, some respondents felt that motivation from the management would boost their morale.
There are limitations to this study. It is worth noting that $\mathrm{KKH}$ is a dedicated standalone women's and children's hospital that does not routinely provide adult emergency medicine or adult non-OBGYN service. Hence, it may not be experiencing the full impact of the COVID-19 pandemic compared to a general hospital. However, the perceptions of our residents may be extrapolated and generalisable to the residents of other specialties. ${ }^{9,10}$ Further research is needed to determine the impact of these implemented measures on clinical training and resident wellness.

In conclusion, our residents have been significantly adversely affected by COVID-19 during the ACGME-I training. As the ACGME-I structure is highly time sensitive, residents preparing for exit examination are especially affected due to the magnitude and prolonged duration of the pandemic. Various interventions to minimise the impact should be considered early, such as the use of online learning platforms and exercising flexibility in promotion requirements. In addition, all hospitals should encourage a supportive work environment, and consult residents in the formulation of key protocols. These measures are crucial to ensure the physical and mental well-being of residents to prevent the effects of stress and burnout.

\section{Acknowledgements}

We would like to acknowledge Dr Victoria Chua, Ms Valencia Zhang, Ms Jamie Thea Jiaying Lin and Ms Choo Xin Hui for their contributions to the interview transcriptions. We would also like to acknowledge Ms Charlene Ong for her assistance in administrative matters and study coordination. 


\section{REFERENCES}

1. Wilder-Smith A, Chiew CJ, Lee VJ. Can we contain the COVID-19 outbreak with the same measures as for SARS? Lancet Infect Dis 2020;20:e102-7.

2. Rambaldini G, Wilson $\mathrm{K}$, Rath $\mathrm{D}$, et al. The impact of severe acute respiratory syndrome on medical house staff: a qualitative study. J Gen Intern Med 2005;20:381-5.

3. Chong MY, Wang WC, Hsieh WC, et al. Psychological impact of severe acute respiratory syndrome on health workers in a tertiary hospital. Br J 2004;185:127-33.

4. Maunder R, Hunter J, Vincent L, et al. The immediate psychological and occupational impact of the 2003 SARS outbreak in a teaching hospital. CMAJ 2003;168:1245-51.

5. Brooks SK, Dunn R, Amlôt R, et al. Thematic review of social and occupational factors associated with psychological outcomes in healthcare employees during an infectious disease outbreak. J Occup Environ Med 2018;60:248-57.

6. Matsuishi K, Kawazoe A, Imai H, et al. Psychological impact of the pandemic (H1N1) 2009 on general hospital workers in Kobe. Psychiatry Clinical Neurosci 2012;66:353-60

7. Sherbino J, Atzema C. "SARS-Ed": severe acute respiratory syndrome and the impact on medical education. Ann Emerg Med 2004;44:229-31.

8. Saunders B, Sim J, Jinks C, et al. Saturation in qualitative research: exploring its conceptualization and operationalization. Qual Quant 2018;52:1893-907.
9. Lai J, Ma S, Wang Y, et al. Factors associated with mental health outcomes among health care workers exposed to coronavirus disease 2019. JAMA Netw Open 2020;3:e203976.

10. Seow J, Lin SX, Khor YP, et al. Stress and Strain in an Orthopaedic Department on the frontlines during the COVID-19 Pandemic: An Analysis of Burnout and the Factors Influencing It. Ann Acad Med Singap 2020;49:814-6.

Jason Lam Shang Leen ${ }^{1} M D$,

Tiffany Tuck Chin Wong ${ }^{* 2}{ }_{M B B S}$, Chee Wai $\underline{\mathrm{Ku}}^{2}{ }_{M D}$, Janine Cynthia Koh ${ }^{1}$ MBBS, Tu Anh Pham $\underline{\text { Nguyen }}{ }^{1} M D$, Raveen Shahdadpuri ${ }^{1}$ MRCPI, Manisha Mathur ${ }^{2}$ MBBS, Cristelle Chow ${ }^{1} M B B S$

\footnotetext{
${ }^{1}$ Department of Paediatrics, KK Women's and Children's' Hospital, Singapore

${ }^{2}$ Department of Obstetrics and Gynecology, KK Women's and Children's

Hospital, Singapore

* Primary co-author
}

Correspondence: Dr Jason Lam Shang Leen, KK Women's and Children's Hospital, 100 Bukit Timah Rd, Singapore 229899.

Email: jason.lam@mohh.com.sg 